\title{
Some Properties of Flows at Freeway Bottlenecks
}

\author{
Lei Zhang and David Levinson
}

\begin{abstract}
The capacity of a freeway segment should be measured only when it is an active bottleneck. The properties of flows at active freeway bottlenecks have a bearing on both the definition of capacity and the procedure of capacity analysis. Past studies have examined the flow features at bottlenecks on several freeways in Toronto, Canada, and San Diego, California. This study examined 27 active bottlenecks in the Twin Cities metro area in Minnesota for a 7-week period. The analysis focuses on the properties of prequeue transition flows $(\mathrm{PQFs})$ and queue discharge flows (QDFs) averaged across various time intervals (30-s, daily average, and long-run average). It is found that the proportion by which flows drop after upstream queues form at all studied bottlenecks ranges from $2 \%$ to $11 \%$. The 30-s QDFs display high variation and should not be assumed to be constant. The daily average QDFs at each studied bottleneck follow a normal distribution based on two normality tests and visual inspection of the normal probability plot. Results also suggest that the long-run average QDFs [mean of 2,016 passenger cars per lane per hour (pcplph)] and PQFs (mean of 2,124 pcplph) are both normally distributed. The implication of these empirical findings on capacity estimation is also discussed.
\end{abstract}

The Highway Capacity Manual (HCM) (1) defines capacity as "the maximum hourly rate at which persons or vehicles reasonably can be expected to traverse a point or a uniform section of a lane or roadway during a given time period under prevailing roadway, traffic, and control conditions." Later, in the glossary chapter, the definition differs slightly and the maximum sustainable flow rate is emphasized. The definition clearly states that the capacity of a freeway element should not be measured when the traffic condition is governed by a bottleneck further downstream. Therefore, capacity should be measured only at, or immediately downstream of, active bottlenecks, not within the queue upstream. Hall and Agyemang-Duah (2) address this issue in detail and conclude that studies examining capacity in the congested region of the fundamental diagram are "based on a mistaken premise about where the data are collected." Therefore, capacity is a parameter dependent only on the flow features at active bottlenecks.

However, current knowledge about the flow features at active bottlenecks suggests that the HCM does not provide a satisfactory method of operationalizing the definition of capacity. Terms appearing in the definition of capacity, such as reasonable expectation and sustainable flows, have not become instrumental in practice. Several studies demonstrate that on the basis of the 15-min criterion for sustainable flows, both the prequeue transition period and the queue

Department of Civil Engineering, University of Minnesota, 500 Pillsbury Drive SE, Minneapolis, MN 55455.

Transportation Research Record: Journal of the Transportation Research Board, No. 1883, TRB, National Research Council, Washington, D.C., 2004, pp. 122-131. discharge period qualify, but the average flow rates during those two periods differ significantly (2-6).

In terms of the numerical values of the capacity at specific freeway segments, the HCM recommends a subtraction method on the basis of the capacity value under ideal conditions. Maximum recorded hourly rates across the nation are summarized, and a flow rate of 2,400 passenger cars per lane per hour ( $\mathrm{pcplph}$ ) is used for freeways with free-flow speeds of 70 to $75 \mathrm{mph}(1 \mathrm{mph}=1.6 \mathrm{~km} / \mathrm{h})$. Then this value is adjusted downward according to various local hindrance and delay factors such as number of lanes, lane width, lateral clearance, weaving configuration, ramp junction, traffic composition, and driver characteristics. The results of the current study strongly suggest that the actual long-run average queue discharge flow (QDF) rates and prequeue transition flow (PQF) rates at various bottlenecks follow separate normal distributions, which leads to the proposition of a new definition of capacity and implies an alternative procedure for capacity analysis.

In past studies on freeway capacity and flow features at freeway bottlenecks, the following characteristics were observed:

1. Flow drop. After a bottleneck activates and a queue forms upstream, the flow at the bottleneck drops and the proportion of the drop ranges from less than $1 \%$ to $10 \%(2-5,7-10)$.

2. QDF. QDFs exhibit near-stationary patterns that slowly alternate at a constant rate and never deviate from the mean more than $5 \%$; sequences of nearly uniform discharge flows gradually change over time $(5,11)$. Another study suggests that the QDFs decrease as the upstream queue gets longer (12). The 5-min QDF rates appear to be nearly normally distributed (2).

3 . Prequeue transition period. The length of the transition period per breakdown ranges from 3 min to $32 \min (2,5)$. The breakdown probability is a function of the mainline flows $(13,14,15)$. The breakdown is always associated with high on-ramp inflows at one site (16).

Most of the cited research is based on traffic data collected on several freeways in Toronto, Ontario, Canada, and San Diego, California. The current study examines the flows at active freeway bottlenecks during a 7-week period in the Twin Cities in Minnesota. Some of those previously observed phenomena are confirmed and others are not. The large data set used in the study also allows the examination of some properties of the flows at freeway bottlenecks not documented in the current body of literature. Some findings further suggest a new way of defining and measuring freeway capacity for operational, design, and planning analyses.

The traffic data and study sites are described next, followed by the methodology for measuring bottleneck flows. The traffic features identified in the study, especially the properties of flows during the prequeue transition and queue discharge periods, are interpreted and 
compared with the results of past studies. The implications of the new findings for freeway capacity are discussed, and conclusions and future research directions are offered.

\section{DATA}

The Minnesota Department of Transportation metropolitan Traffic Management Center operates an extensive freeway management system in the Twin Cities. Thirty-second traffic volume and occupancy data from more than 4,000 single-loop detectors are available on more than $300 \mathrm{~km}$ of freeways. Loop detectors were usually installed at $0.8-\mathrm{km}$ (half-mile) intervals, but additional detectors were installed at most merging and diverging areas such that traffic data were directly measurable for each individual segment with uniform flow characteristics. Data for the afternoon peak period (13:00 to 21:00) from October 16 to December 3, 2000, were used since all ramp meters were shut off during those 7 weeks for an evaluation study mandated by the 2000 session of the Minnesota legislature.

The study was limited to normal weekdays so that the driver population at each bottleneck would be relatively constant and familiar with the facility. The volume and occupancy data collected during the shutoff period were carefully tested, and eight types of corrupt detectors were identified and eliminated from the analysis according to the findings of several previous studies (17-19). Finally, in order to minimize the impact of normal detection noise, 10-min moving averages with 30-s successive intervals of raw 30-s flow and occupancy data were computed and used in the following analysis. This data set was also used in a subsequent comparison study for the metering-off scenario (6), but that research focused on the impacts of ramp metering on freeway capacity.

Twenty-seven freeway sections with frequent occurrences of breakdown were examined because reliable traffic data were only available at these locations after various tests for corrupt detectors. Their locations in the Twin Cities freeway system are shown in Figure 1. The free-flow speed at all selected segments exceeds $96 \mathrm{~km} / \mathrm{h}$ (60 mph). The detector deployment and geometric characteristics of each individual bottleneck are illustrated in Figure 2. Various types of bottlenecks can be found in the final sample, including busy entrance ramps, weaving sections, lane drops, tunnels, bridges, horizontal curves, vertical curves, and combined curves. The least congested bottleneck of all 27 experienced only six breakdowns during the study period, whereas at one location, more than 70 breakdowns were observed. Heavy-vehicle percentages were collected at all studied bottlenecks in 2000; they ranged from $0.7 \%$ to $8.8 \%$. Heavy-vehicle adjustments are based on passenger-car equivalents given in the HCM.

Adverse weather conditions, road construction and maintenance, and traffic accidents were identified, and the days under these conditions were excluded from the analysis at affected bottleneck locations. The capacity reduction associated with construction and maintenance work is typically due to lane closure. Days with bad weather or laneblocking accidents were not included because it is impossible for engineers to control them. The capacity analysis in response to those three factors should follow specific procedures and is beyond the scope of this study.

\section{MEASURING BOTTLENECK FLOWS}

Breakdown is a freeway traffic phenomenon usually associated with a sharp speed drop and possibly a flow drop at bottlenecks during a high-demand period. After breakdown, a queue will form upstream.
Therefore, an active bottleneck is characterized by a queue upstream and unrestricted flow condition downstream (20, pp. 133-135, 259). Flows were measured and studied during two periods: prequeue transitions and queue discharges. Correct measurements of those two flows require a reliable method to identify the starting and ending times of both the prequeue transition period and the queue discharge period for each breakdown occurrence. Several methods using time-series occupancy and speed data were summarized by Daganzo (20, pp. 133-135, 259). Cassidy and Windover (21) propose a diagnostic tool based on wave propagation using transformed cumulative count and occupancy curves. Hall and Agyemang-Duah (2) base their analysis on the ratio of occupancy to flow. Purely visual inspection was also used in previous studies to identify breakdowns $(14,22)$. Das and Levinson (23) combined queuing and statistical analyses to examine bottleneck formation. After experiments at several locations, a method based on two occupancy threshold values was used in this study. Any freeway detection station at a specific time interval can be categorized according to the two threshold values into three conditions: congested, uncongested, and intermediate.

A freeway mainline detection station usually consists of multiple detectors, each corresponding to one lane. If in a data collection interval (30 s), the minimum occupancy reading from all detectors at a station is larger than $25 \%$ (about $39 \mathrm{veh} / \mathrm{lane} / \mathrm{km}$ with $5 \%$ trucks), the traffic at the station is in the congested region. If the maximum occupancy reading from all detectors is smaller than $20 \%$ (31 veh/ lane $/ \mathrm{km}$ ), the station is considered to be uncongested. If neither condition is satisfied, the station is in the intermediate period. The two occupancy threshold values were obtained after experiments in which congestion predicted by the two thresholds was compared with visual inspection results.

All freeways in the Twin Cities traffic management systems were divided into about 300 segments, each of which had an upstream and a downstream detector station. Of hundreds of candidates, only 27 freeway segments, in which detector data survived the eight validity tests, were repeatedly observed to be active bottlenecks. If the upstream station of a freeway segment is congested and the downstream station is uncongested for more than $5 \mathrm{~min}$, a breakdown has just occurred and the segment is considered an active bottleneck. The beginning interval of that 5 -min period is also the starting time of a queue discharge period $\left(t_{s}\right)$. After a certain amount of time, the bottleneck will no longer be active and the upstream queue will dissipate completely. Once both the upstream and the downstream stations of the segment operate under the uncongested condition for more than $5 \mathrm{~min}$ after a queue discharge period, the bottleneck recovers, and the start of that 5-min period determines the ending time of the queue discharge period $\left(t_{e}\right)$. If the downstream station of a freeway section is congested, the traffic condition at the current section is determined by bottlenecks further downstream. These observations were excluded from the analysis of the current freeway section. The 30-s, daily average, and long-run average QDFs can be calculated on the basis of the readings from the downstream station of the bottleneck once the duration of each bottleneck activation is identified (see Figure 3, Periods 4 and 6).

The method with two occupancy thresholds and the adoption of minimum-maximum criteria together constitutes a conservative algorithm for breakdown identification and guarantees that the QDFs are not collected during periods when the bottleneck is not active. It also introduces an intermediate period (Period 3 in Figure 3). Breakdowns occur and queues build up from one lane to all lanes during the intermediate periods. It was found that the duration of intermediate periods is usually very short (less than 10 min per breakdown). 


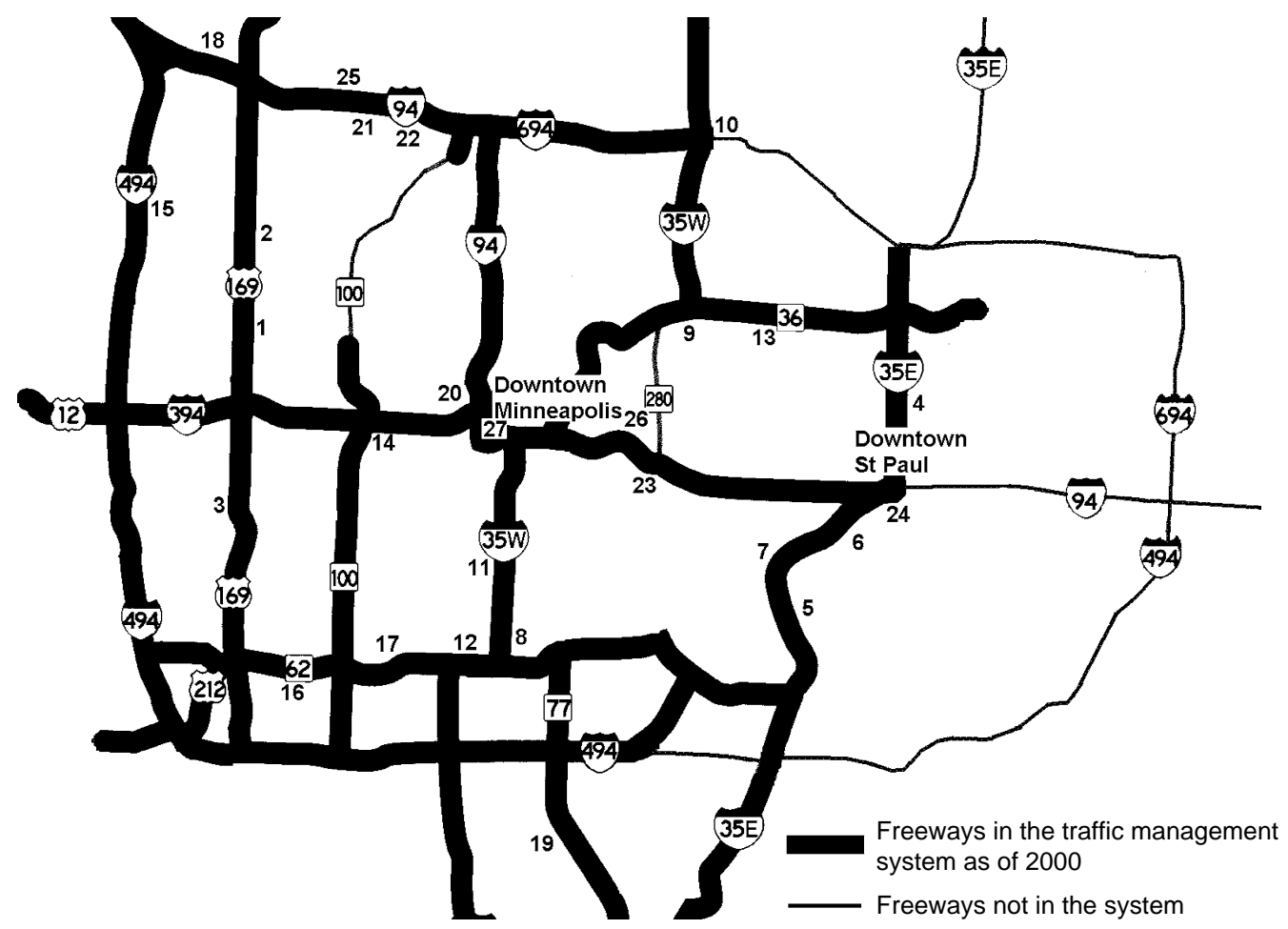

\begin{tabular}{llll}
\hline Id & Freeway & Location & Type \\
\hline 1 & TH169 NB & TH55 & Weaving section \\
2 & TH169 NB & Medicine Lake Rd & Entrance ramp \\
3 & TH169 SB & Cedar Lake Rd & Entrance ramp \\
4 & I-35E NB & Arlington Ave & Entrance ramp \\
5 & I-35E NB & Shepard Rd & Bridge \\
6 & I-35E NB & Ramsey-Grand & Entrance ramp \\
7 & I-35E SB & St Clair Ave & Entrance ramp \\
8 & I-35W NB & 50th St & Entrance ramp \\
9 & I-35W NB & TH36 & Weaving section \\
10 & I-35W NB & I-694 & Weaving section \\
11 & I-35W SB & 46th St & Entrance ramp \\
12 & I-35W SB & TH62 & Weaving section, Horizontal curve \\
13 & TH36 EB & Lexington Ave & Entrance ramp \\
14 & I-394 EB & TH100 & Entrance ramp \\
15 & I-494 NB & 49th Ave & Vertical curve, Entrance ramp \\
16 & TH62 EB & Tracy Ave & Horizontal curve, Entrance ramp \\
17 & TH62 WB & Valley View Rd & Horizontal curve, Entrance ramp \\
18 & I-694 WB & CR61 & Entrance ramp \\
19 & TH77 SB & Old Shakopee Rd & Bridge, Entrance ramp \\
20 & I-94 EB & Hennepin Ave & Tunnel, 3-d curve, Entrance ramp \\
21 & I-94 EB & CR81 & Horizontal curve, Entrance ramp \\
22 & I-94 EB & CR152 & Entrance ramp \\
23 & I-94 EB & TH280 & 3-d curve, Entrance ramp \\
24 & I-94 EB & I-35E & Weaving section \\
25 & I-94 WB & CR81 & Lane drop 3 to 2 \\
26 & I-94 WB & Riverside Ave & 3-d curve, Entrance ramp \\
27 & I-94 WB & Hennepin Ave & Tunnel, 3-d curve \\
\hline & & &
\end{tabular}

FIGURE 1 Location of studied bottlenecks.

Therefore, the intermediate periods were simply excluded from the following analysis of bottleneck flows. This treatment slightly reduced the length of the computed queue discharge period and possibly the prequeue transition period too but should not have biased the flow measurements during both periods in any significant way.
When a prequeue transition period (Period 2 in Figure 3) turns into an intermediate period or the flow rate drops below the long-run average QDF rate without a subsequent breakdown (Period 1 in Figure 3) at time $\tau_{e}$, the prequeue transition period ends. It is more controversial to identify the starting time of the prequeue transition period $\left(\tau_{s}\right)$. Several past studies provide different definitions for the 

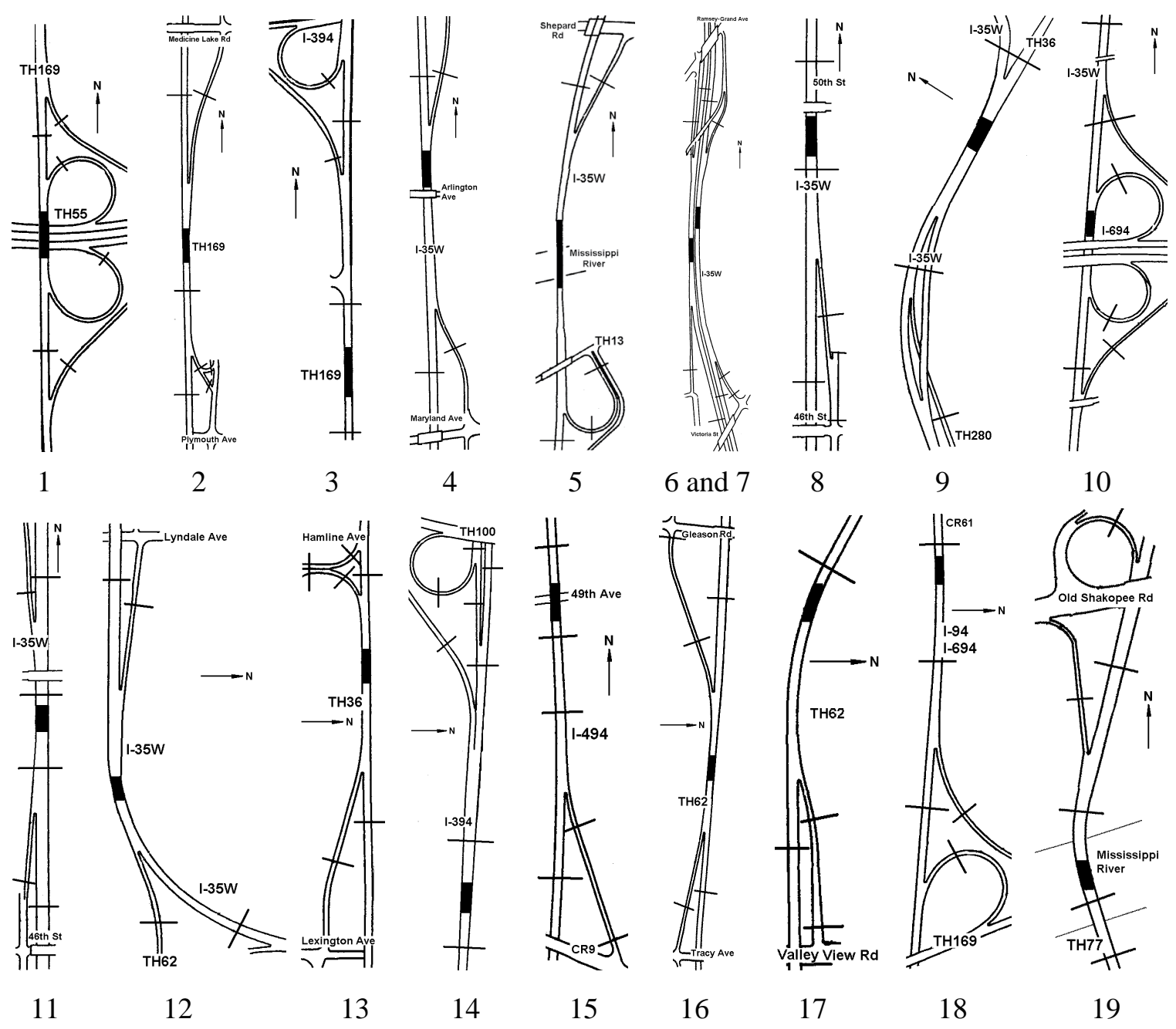

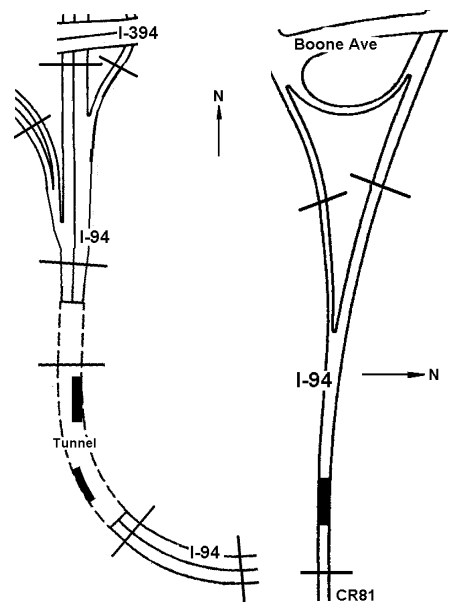

20 and 27

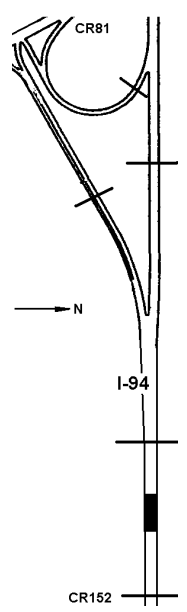

22

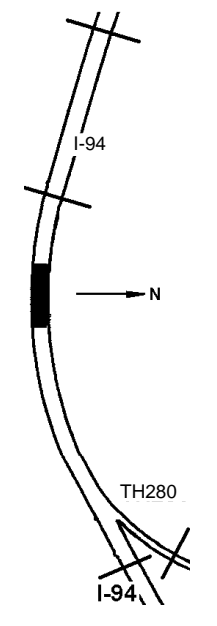

23

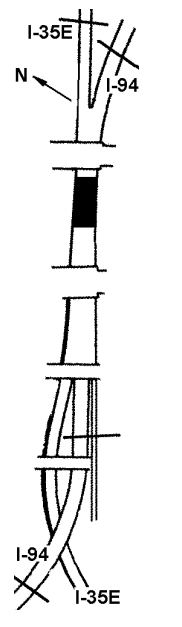

24

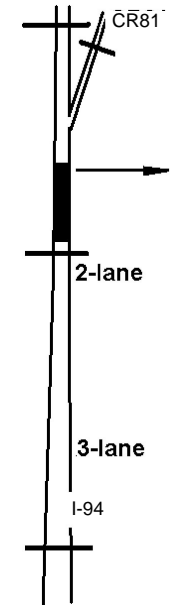

25

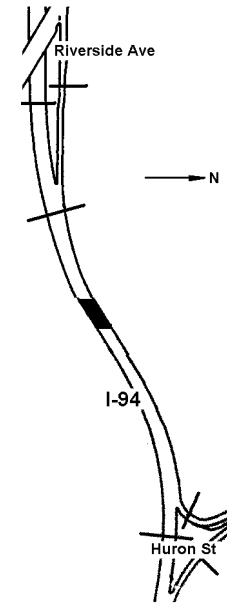

26

Bottleneck

Detector Station

FIGURE 2 Detector deployment and geometry characteristics at studied bottlenecks. 


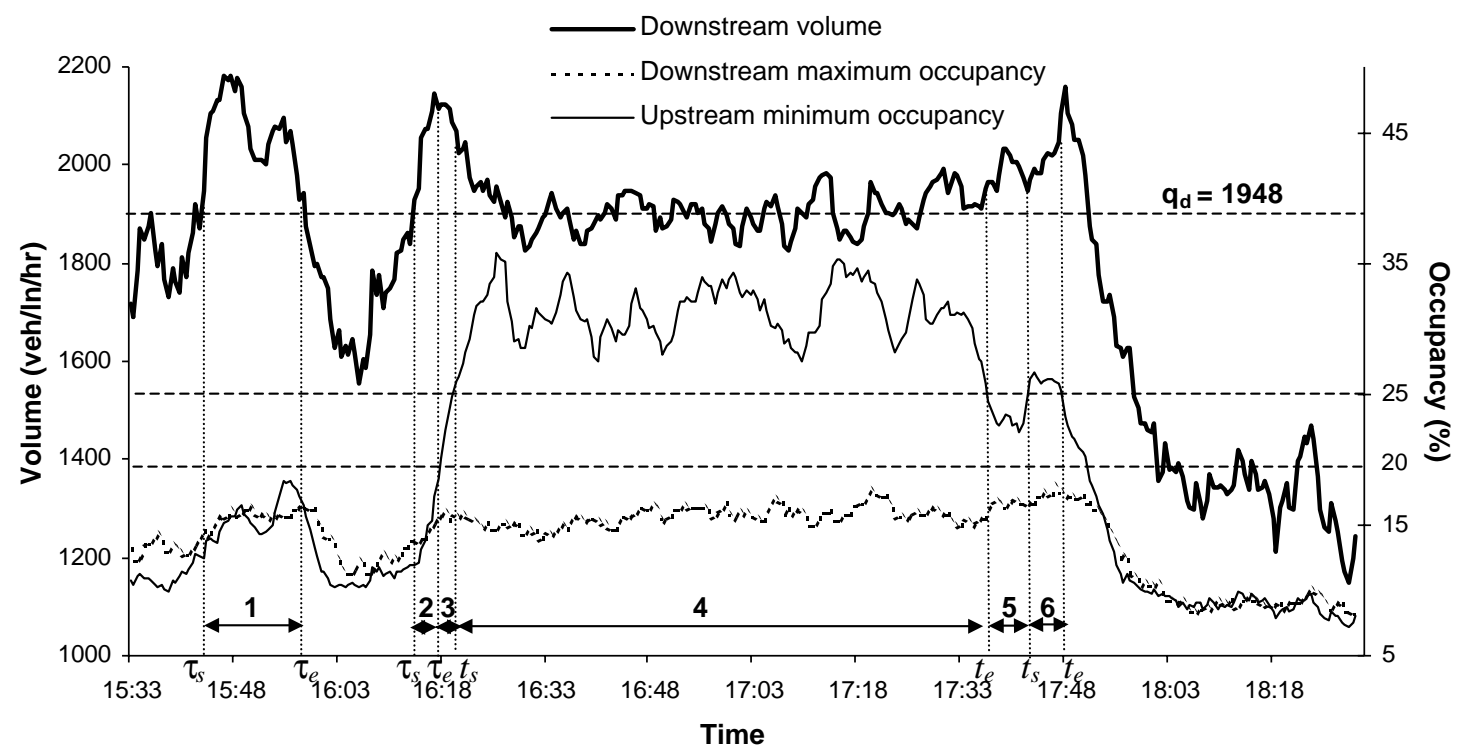

FIGURE 3 Application of method with two occupancy thresholds to Bottleneck 5, Nov. 21, 2000 [q $q_{d}$ : long-run average queue discharge flow rate calculated from the 7-week study period (or 1,948 pcplph); 1 . first observed prequeue transition period (15 min, not followed by a breakdown); 2 . second prequeue transition period ( $4 \mathrm{~min}$, followed by a breakdown); 3 . intermediate period excluded from the analysis ( $3 \mathrm{~min}$ ); 4 . first queue discharge period ( $1 \mathrm{~h}, 17 \mathrm{~min}) ; 5$. a period lost due to conservative occupancy thresholds (5 $\mathrm{min}) ;$ and 6. another queue discharge period $[5 \mathrm{~min})]$.

prequeue transition period and therefore use different criteria to find $\tau_{s}(2,5,14)$. The flows during the prequeue transition period could be high enough to result in a subsequent breakdown. In other words, the breakdown probability during the transition period should be significantly larger than zero.

Without a thorough understanding of the breakdown probability function at the study sites, the long-run average QDF at a bottleneck should serve as a good indicator of the starting time of a prequeue transition period. Therefore, $\tau_{s}$ is determined by the interval in which the flow at a freeway section exceeds its long-run QDF and both upstream and downstream stations are uncongested. It should be noted that a prequeue discharge period may not always be followed by a queue discharge period. The flow rates can be very high for a while and then drop without a breakdown simply by chance. The $30-\mathrm{s}$, daily average, and long-run average transition flows that can be obtained after the starting and the ending times of each transition period are identified. Figure 3 provides a complete example of how $t_{s}, t_{e}, \tau_{s}$, and $\tau_{e}$ were determined from the traffic data.

Sometimes a bottleneck is activated because of insufficient offramp capacity (a diverge bottleneck), which can be identified by high occupancies (larger than 25\%) at the corresponding off-ramp detector station. Diverge bottlenecks were eliminated from the analysis because the QDF rates at these bottlenecks are mainly determined by the off-ramp capacity, not the characteristics of the freeway mainline segment.

\section{RESULTS}

The properties of flows at the 27 bottlenecks were examined for a minimum of 24 days and a maximum of 28 days during the 7-week study period (see Table 1). This variation is primarily due to the different weather and roadway conditions across all study sites. The least congested bottleneck (Id 19) experienced only six breakdowns during the study period, whereas more than 70 breakdowns were observed at one heavily congested location (Id 1). The summary statistics of the QDFs and the PQFs will be presented first followed by their statistical distributions.

The long-run average QDFs at all studied bottlenecks were computed from all days during the study period; the mean was 2,016 pcplph, and the standard deviation was 141 pcplph after heavy vehicles were controlled for. The long-run average PQFs have a mean of 2,124 pcplph and a standard deviation of $128 \mathrm{pcplph}$. That the flow drops after the formation of upstream queues at bottlenecks was confirmed by the results. The average percentage of flow drop at each bottleneck was calculated on the basis of the QDFs and the PQFs from all breakdown observations. After breakdown, the average flow drops at all sites between $2 \%$ and $11 \%$. The mean is about $5 \%$ and the standard deviation is $2 \%$. The flow drops at all locations are statistically significant at the 0.01 level. The average daily duration of the queue discharge periods at all bottlenecks is about $38 \mathrm{~min}$, and the average prequeue transition period is as long as $68 \mathrm{~min}$. This finding contrasts with the results from past studies, which found that the prequeue transition period is usually less than $30 \mathrm{~min}$. The major reason for the discrepancy is that the prequeue transition periods not followed by a breakdown were included in this study but not considered previously. A freeway segment can operate at a flow rate higher than its long-run queue discharge rate for a significant amount of time and not incur a subsequent breakdown. This phenomenon was repeatedly observed at all study sites, and Period 1 in Figure 3 is an example. The correlation between the duration of the queue discharge period and the duration of the prequeue transition period was further examined, and it was found that the two are negatively correlated, with a correlation coefficient of -0.26 . The interpretation is that, in general, more heavily congested bottlenecks have shorter prequeue transition periods. 
TABLE 1 Summary Statistics of QDFs and Prequeue Flows at Studied Bottlenecks

\begin{tabular}{|c|c|c|c|c|c|c|c|c|c|c|}
\hline Id & $\begin{array}{r}\text { Days of } \\
\text { Analysis }\end{array}$ & $\begin{array}{r}\text { No. of } \\
\text { Breakdowns }\end{array}$ & $\begin{array}{l}\text { No. of } \\
\text { Lanes }\end{array}$ & $\begin{array}{l}\text { Heavy Veh. } \\
\text { Percentage }\end{array}$ & $\begin{array}{r}\text { Avg. QDF } \\
\text { (pcplph) }\end{array}$ & $\begin{array}{r}\text { Avg. QDF Period } \\
\text { (hr:mm/day) }\end{array}$ & $\begin{array}{l}\text { Avg. Prequeue } \\
\text { Flow (pcplph) }\end{array}$ & $\begin{array}{r}\text { Avg. Prequeue } \\
\text { Period (hr:mm/day) }\end{array}$ & $\begin{array}{l}\text { Percentage } \\
\text { Flow Drop }\end{array}$ & $\begin{array}{l}\text { V of Daily } \\
\text { QDF Dist. }\end{array}$ \\
\hline 1 & 24 & 74 & 2 & $4.0 \%$ & 2135 & $0: 50$ & 2178 & $0: 13$ & $2 \%$ & 0.04 \\
\hline 2 & 26 & 55 & 2 & $4.3 \%$ & 2055 & $1: 07$ & 2175 & $1: 08$ & $6 \%$ & 0.04 \\
\hline 3 & 27 & 8 & 2 & $4.0 \%$ & 2111 & $0: 10$ & 2187 & $0: 27$ & $4 \%$ & 0.03 \\
\hline 4 & 28 & 9 & 3 & $4.2 \%$ & 2019 & $0: 15$ & 2195 & $1: 54$ & $9 \%$ & 0.02 \\
\hline 5 & 27 & 21 & 2 & $3.0 \%$ & 1948 & $0: 47$ & 2069 & 1:08 & $6 \%$ & 0.02 \\
\hline 6 & 26 & 30 & 2 & $3.1 \%$ & 1992 & $0: 38$ & 2051 & $0: 22$ & $3 \%$ & 0.05 \\
\hline 7 & 27 & 11 & 2 & $0.7 \%$ & 2019 & $0: 14$ & 2100 & $0: 44$ & $4 \%$ & 0.04 \\
\hline 8 & 28 & 26 & 3 & $4.1 \%$ & 1953 & $0: 27$ & 2111 & $2: 59$ & $8 \%$ & 0.03 \\
\hline 9 & 27 & 11 & 4 & $5.2 \%$ & 2160 & $0: 20$ & 2290 & $0: 36$ & $6 \%$ & 0.05 \\
\hline 10 & 27 & 56 & 3 & $5.7 \%$ & 2332 & $1: 52$ & 2386 & 0:04 & $2 \%$ & 0.04 \\
\hline 11 & 26 & 19 & 3 & $4.1 \%$ & 2281 & $0: 12$ & 2350 & $1: 13$ & $3 \%$ & 0.04 \\
\hline 12 & 27 & 20 & 3 & $5.1 \%$ & 1810 & $0: 38$ & 1895 & 3:05 & $5 \%$ & 0.05 \\
\hline 13 & 27 & 14 & 2 & $3.4 \%$ & 2154 & $0: 14$ & 2268 & $0: 40$ & $5 \%$ & 0.03 \\
\hline 14 & 27 & 70 & 3 & $3.3 \%$ & 1772 & $0: 59$ & 1859 & 1:04 & $5 \%$ & 0.03 \\
\hline 15 & 26 & 33 & 2 & $7.4 \%$ & 2190 & $0: 50$ & 2242 & $0: 14$ & $2 \%$ & 0.04 \\
\hline 16 & 24 & 28 & 2 & $2.6 \%$ & 2038 & $0: 21$ & 2168 & $0: 38$ & $6 \%$ & 0.02 \\
\hline 17 & 26 & 14 & 2 & $2.7 \%$ & 1974 & $0: 19$ & 2051 & $1: 08$ & $4 \%$ & 0.04 \\
\hline 18 & 23 & 51 & 2 & $8.8 \%$ & 2053 & $1: 59$ & 2152 & $0: 53$ & $5 \%$ & 0.04 \\
\hline 19 & 27 & 6 & 3 & $2.6 \%$ & 2077 & $0: 19$ & 2178 & $0: 49$ & $5 \%$ & 0.06 \\
\hline 20 & 28 & 8 & 3 & $4.5 \%$ & 1862 & $0: 29$ & 1947 & $1: 43$ & $5 \%$ & 0.03 \\
\hline 21 & 26 & 43 & 2 & $8.0 \%$ & 1938 & $0: 28$ & 2040 & 1:05 & $5 \%$ & 0.04 \\
\hline 22 & 28 & 21 & 2 & $7.5 \%$ & 1888 & $0: 28$ & 2060 & $2: 36$ & $9 \%$ & 0.05 \\
\hline 23 & 27 & 61 & 3 & $5.1 \%$ & 2093 & $0: 54$ & 2191 & $0: 49$ & $5 \%$ & 0.03 \\
\hline 24 & 24 & 42 & 4 & $5.3 \%$ & 1902 & $1: 06$ & 2109 & $0: 58$ & $11 \%$ & 0.04 \\
\hline 25 & 28 & 37 & 2 & $8.2 \%$ & 1994 & $0: 30$ & 2126 & $1: 33$ & $7 \%$ & 0.04 \\
\hline 26 & 28 & 7 & 3 & $5.2 \%$ & 1798 & $0: 16$ & 1953 & $1: 56$ & $9 \%$ & 0.02 \\
\hline 27 & 28 & 34 & 3 & $4.5 \%$ & 1883 & $0: 27$ & 2010 & 1:03 & $7 \%$ & 0.04 \\
\hline
\end{tabular}

$\mathrm{CV}=$ coefficient of variance.

The daily average and 30-s QDFs at each bottleneck were also examined. The 30-s QDFs at most bottlenecks display very high variability. The ratio of the range to the mean of those 30-s flows can be as high as $50 \%$. Though most of the 30 -s flows cluster in a region not very far from the mean $( \pm 15 \%)$, the results clearly show that the QDFs during short time intervals do not follow a uniform or even a near-uniform distribution. This finding implies that even during the queue discharge period, disturbances would emanate away from the bottleneck. Those disturbances propagating backward may be amplified and cause fast- and slow-moving queues.

Hall and Agyemang-Duah (2) observed that the 5-min QDFs at one bottleneck west of Toronto appeared to follow a near-normal distribution. In the current study, three normality diagnostic tools were used: the normal probability plot, Shapiro-Wilk normality test (24), and skewness-kurtosis test. Since each of the two normality tests were critiqued for the inability to reject some specific nonnormal patterns, a normality hypothesis will be rejected if either one of the two tests rejects it. If a normality hypothesis passes both tests, the normal probability plot will be provided for an additional visual inspection. The assumption that the 30-s QDFs are normally distributed was rejected by both tests at the 0.05 level at all bottlenecks primarily because of heavy tails at both ends. Therefore, one cannot assume that the QDFs during short time intervals follow a normal distribution either. The actual distributions of those 30-s QDFs depend on the real-time traffic composition, driver characteristics, and demand pattern of an upstream entrance ramp. It is not clear what kind of distribution best describes the variation of the 30-s QDFs, but better understanding of the distribution of the QDFs in short time intervals would improve freeway queuing models.
The properties of the average QDFs during longer periods are more important than those during short periods since they have implications for the definition of capacity and the procedure of capacity analysis. The central limit theorem suggests that regardless of the distribution of the 30-s flows, QDFs tend to follow a normal distribution once averaged across a long period. So it is reasonable to suspect that the daily average QDFs at each bottleneck are normally distributed. These hypotheses were again tested and the normality assumption can be rejected at only one location (Bottleneck 22) by the Shapiro-Wilk test at the 0.05 level. The normal probability plots of the daily average QDFs of all bottlenecks are given in Figure 4. The plots and the test results provide strong evidence that daily average QDFs at individual freeway bottlenecks are normally distributed.

The variation of the normal distribution should be explained by the day-to-day variability of demand patterns, traffic composition, driver population, percentage flows from ramps, and weaving volumes of different types as suggested by the HCM. But in general, the variation of the daily average QDFs at each bottleneck is not very large. The coefficient of variation (standard deviation divided by the mean) never exceeds 0.05 (the last column in Table 1). Given a normal distribution, this finding means that even at the bottleneck with the highest day-to-day variation, the daily average QDF is within $\pm 5 \%$ of the long-run average QDF for about $68 \%$ of the time and within $\pm 10 \%$ for about $95 \%$ of the time. However, variations of this magnitude in QDFs can have major impacts on travel time and queue extent.

An interesting finding of this study is the properties of the longrun average QDFs and PQFs at different bottlenecks. How these two parameters vary from location to location (and why) has an important meaning for capacity analysis and hence the design of freeway 


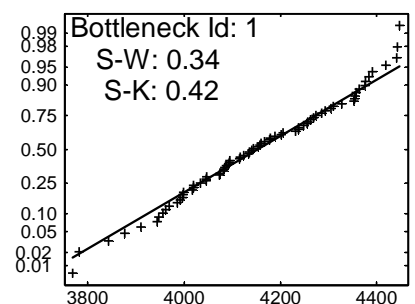

(a)

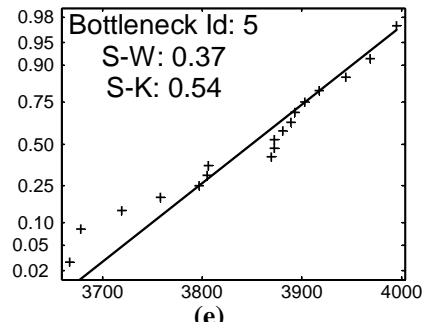

(e)

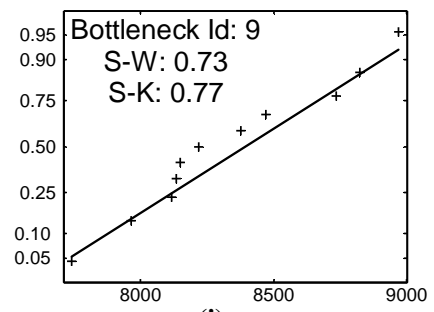

(i)
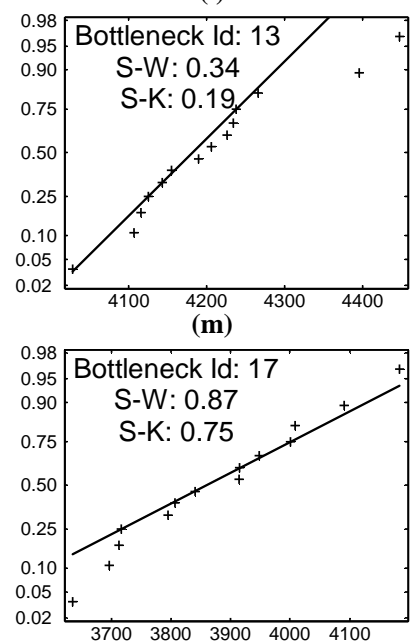

(q)

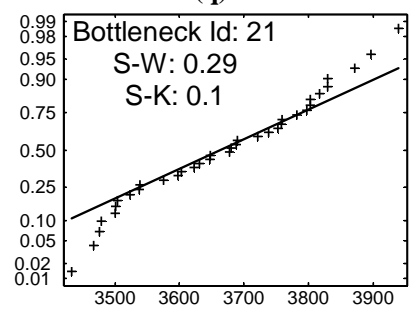

(u)

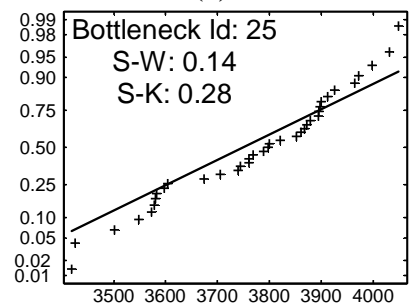

(y)

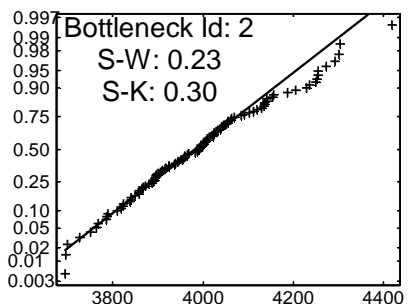

(b)

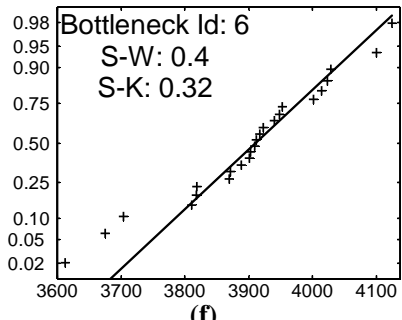

(f)

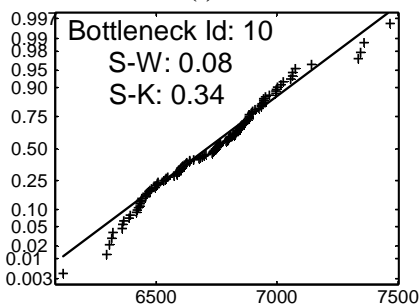

(j)

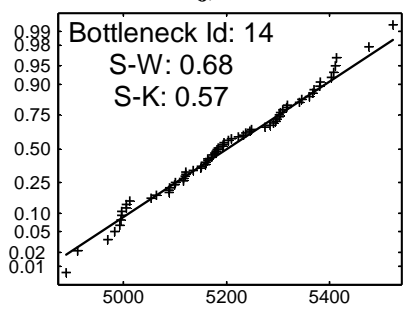

(n)

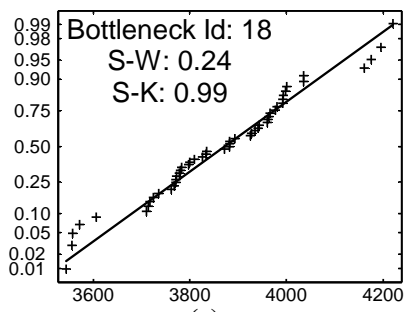

(r)

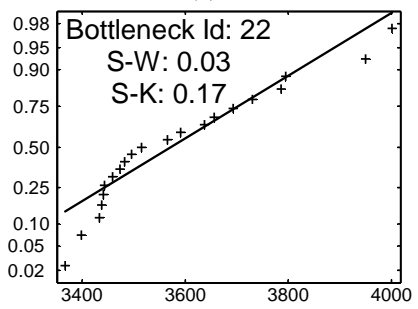

(v)

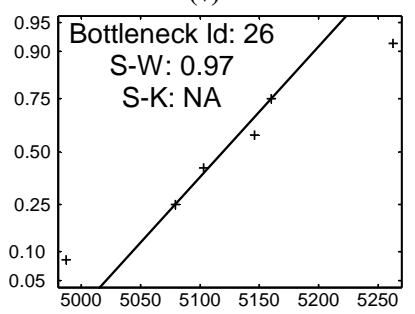

(z)

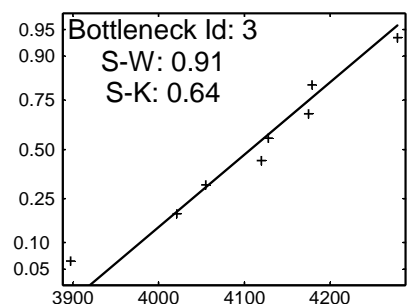

(c)

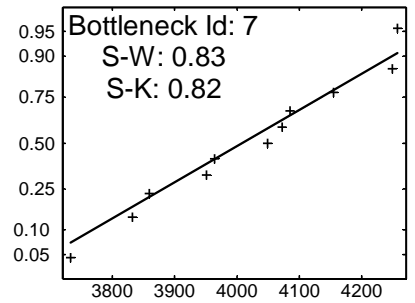

(g)

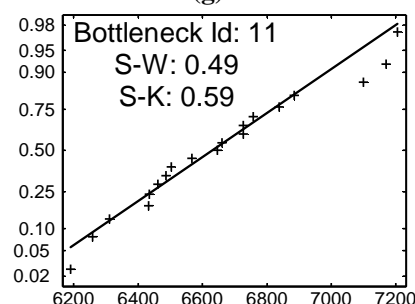

(k)

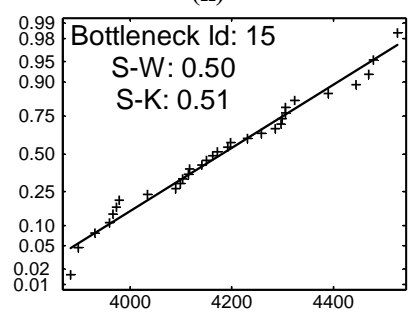

(o)

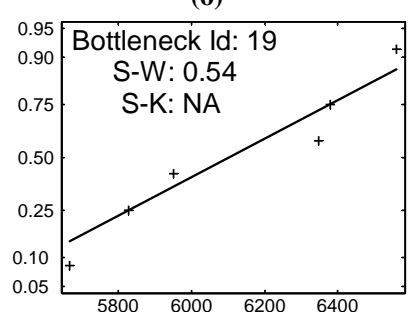

(s)

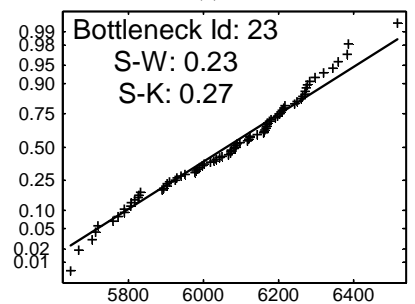

(w)

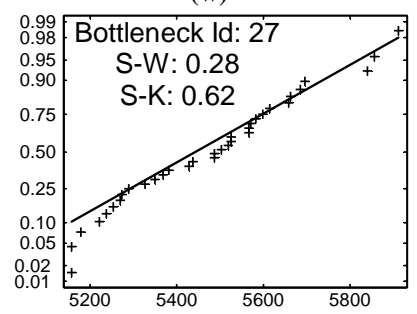

(aa)

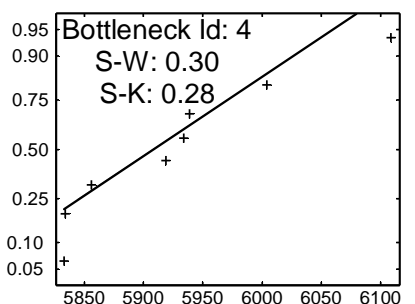

(d)

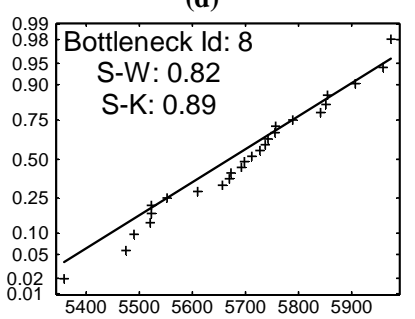

(h)

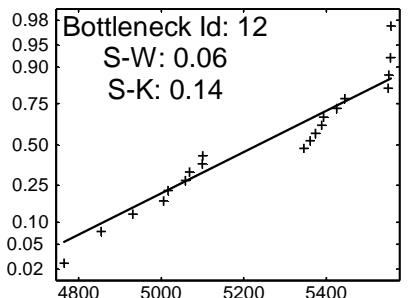

(I)
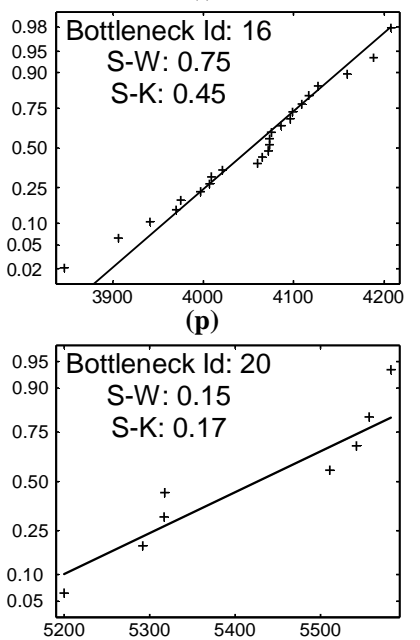

(t)

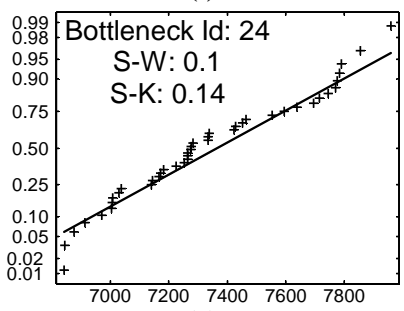

(x)

Y-axis: Probability

X-axis: Flows (All lanes)

Note: Numbers reported in the graphs are test $P$ values.

NA: Test not applicable due to small sample size.

FIGURE 4 Normality test: average QDF rate per breakdown (S-W = Shapiro-Wilk test; S-K = skewness-kurtosis test). 
facilities. When all 27 observations of long-run average QDFs and PQFs are examined, the hypothesis that they follow normal distributions cannot be rejected at the 0.05 level on the basis of the results of the Shapiro-Wilk and skewness-kurtosis normality tests. Further examination shows that the observations of long-run average QDFs and PQFs conform with the theoretical normal probability plots very well (see Figure 5). These results suggest that both long-run average QDFs and long-run average PQFs at various bottlenecks are normally distributed. This normality cannot be explained by the central limit theorem because the long-run average QDFs and PQFs are from different distributions (each bottleneck has a different daily average QDF distribution). The normality and the variation cannot be explained by traffic composition either because after the adjustment for heavy vehicles, the two variables become even more "normal," as seen in the normality plots without any reduction in standard deviation.

The HCM suggests some other factors that may explain the variation of capacity at different freeway segments, such as number of lanes, lane width, horizontal and vertical alignments, the existence of an upstream entrance ramp, and waving section characteristics. A simple linear regression model with QDF as the dependent variable (PQF is highly correlated with $\mathrm{QDF}$ - the correlation coefficient is 0.96 - so a separate model for PQF is not examined) was estimated using those parameters as predictors. It was found that variables such as number of lanes, horizontal curvature, and grade have negative coefficients. However, none of the coefficients was statistically significant at the 0.05 level, and all predictors contribute to only $25 \%$ of the total variance. It is the objective of an ongoing study to explain the variation of the long-run average QDFs and PQFs with more independent variables and more-sophisticated statistical models.

\section{CAPACITY}

During the past two decades there has been a gradual increase in the HCM-recommended freeway capacity value under ideal conditions. The 1985 version suggests 2,000 pcplph for freeways with design speeds of $70 \mathrm{mph}$ and $60 \mathrm{mph}(1 \mathrm{mph}=1.6 \mathrm{~km} / \mathrm{h})$ and $1,900 \mathrm{pcplph}$ for $50 \mathrm{mph}$. The 1994 update raises the values to 2,200 pcplph for two-lane freeways and 2,300 pcplph for freeways with three or more lanes. The most recent millennium edition recommends $2,400 \mathrm{pcplph}$ for freeways with free-flow speeds of 70 and $75 \mathrm{mph}$ and 2,300 pcplph for freeways with free-flow speeds of $65 \mathrm{mph}$ as the capacity under

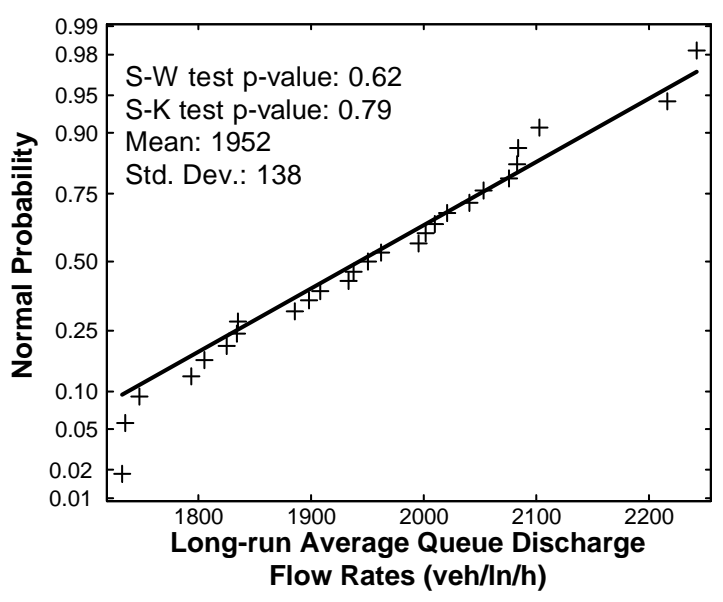

(a)

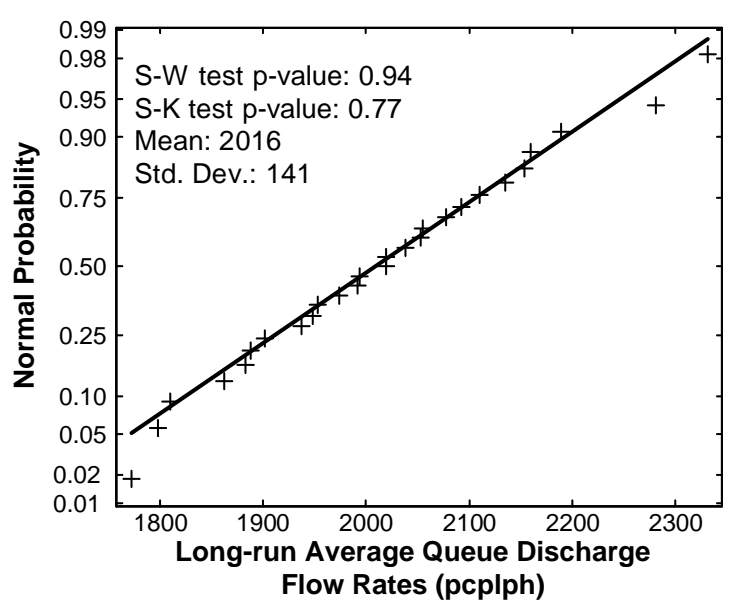

(c)

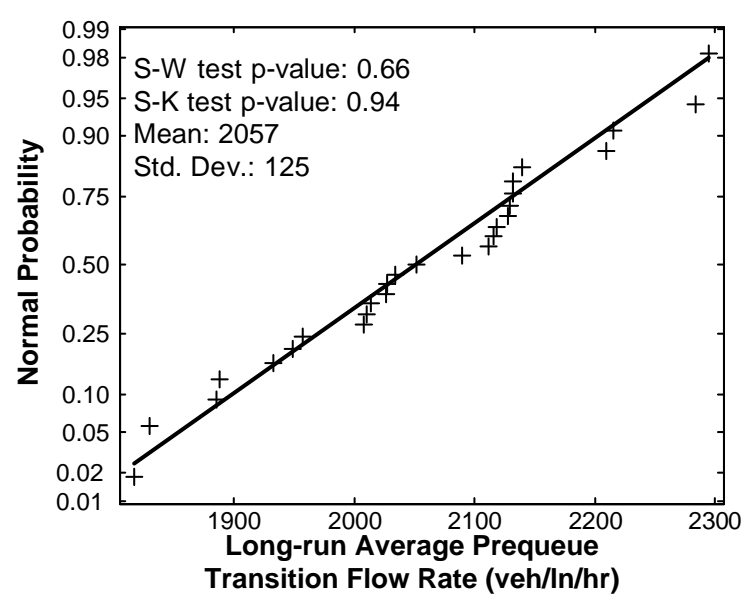

(b)

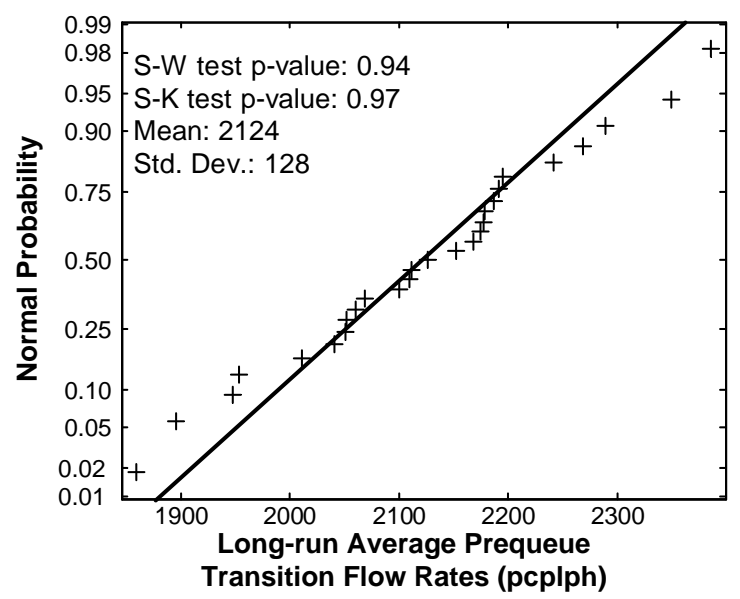

(d)

FIGURE 5 Normality test: long-run average QDF rates and PQF rates leach point representing long-run average QDF rate or PQF rate at a bottleneck): $(a, b)$ before heavy-vehicle adjustment and $(c, d)$ after heavy-vehicle adjustment. 
base conditions. Capacity is usually operationalized as the highest 15-min flow rate observed at a facility.

The long-run average QDFs of the 27 uniform freeway segments examined in this study range from 1,772 to $2,332 \mathrm{pcplph}$, and the long-run average PQFs range from 1,859 to 2,386 pcplph. These results support the increase in the capacity value under the base condition since 2,300 and 2,400 pcplph correspond to the upper tail of the distributions of both flow rates. However, the increase in capacity under ideal conditions is acceptable only because the current procedure of capacity analysis in the HCM is a downward-adjustment process, which requires a base value high enough to avoid systematic underestimation.

Past studies have also discussed which flow rate should be considered as the capacity of a freeway element, QDF or PQF. The lengths of the two flow periods during a typical peak period both significantly exceed $15 \mathrm{~min}$ according to the results. The results further suggest that the long-run average QDFs and PQFs at various locations follow two different normal distributions. The difference of the means is about $100 \mathrm{pcplph}$. The values of these two flows and their duration at a specific freeway bottleneck determine the performance of that bottleneck under any demand condition. Therefore, as a general definition equation, the capacity $(C)$ of a freeway segment should be a weighted sum of the two flow rates:

$C=\mathrm{QDF} \cdot \theta+\mathrm{PQF} \cdot(1-\theta) \quad(0 \leq \theta \leq 1)$

If the capacity is measured as the highest 15 -min flow rate, $\theta$ is likely to be zero and effectively a value somewhere in the upper tail of the $\mathrm{PQF}$ distribution is used, which is typically much higher than both the long-run average QDF and the long-run average PQF. For instance, Period 1 in Figure 3 provides a very high 15-min flow rate (almost 2,100 veh/ln/h), but the actual long-run average QDF and PQF are only 1,908 and $2,026 \mathrm{veh} / \mathrm{ln} / \mathrm{h}$, respectively, at the bottleneck.

Some researchers suggest that the QDF should be considered as the capacity because it is stable and can be reached repeatedly from day to day. In that case, $\theta$ would be 1 . If a simple, consistent, and unique capacity value for each individual freeway segment is the goal, the value of $\theta$ may be calculated on the basis of the relative duration of the queue discharge period and the prequeue transition period. According to the results, $\theta$ is 0.36 on average (time-weighted) but varies from location to location.

Alternatively, how capacity should be estimated and the value of $\theta$ be determined can depend on the purpose of specific applications. As a reviewer suggests, for planning purposes, analysts may want to use PQF to denote the highest flow that can be sustained before breakdown in order to ensure that the value is not exceeded in planning analyses.

Putting aside the weights and focusing on the distribution of capacity at various bottlenecks, as long as the long-run average QDFs and PQFs are normally distributed, the capacity must also be normally distributed according to Equation 1. The means and the standard deviations of the QDFs and PQFs define the bounds of the mean and the standard deviation of the capacity distribution (see Figure 6). The actual mean of the capacity distribution also depends on the value of $\theta$, and the standard deviation also depends on the covariance of the QDFs and the PQFs. Nevertheless, no matter what the covariance and the weights are, the capacity at various bottlenecks also follows a normal distribution.

This conclusion may have some implications for the procedure of capacity analysis because it reveals that capacity values of most freeway bottlenecks cluster in the middle of the normal distribution.

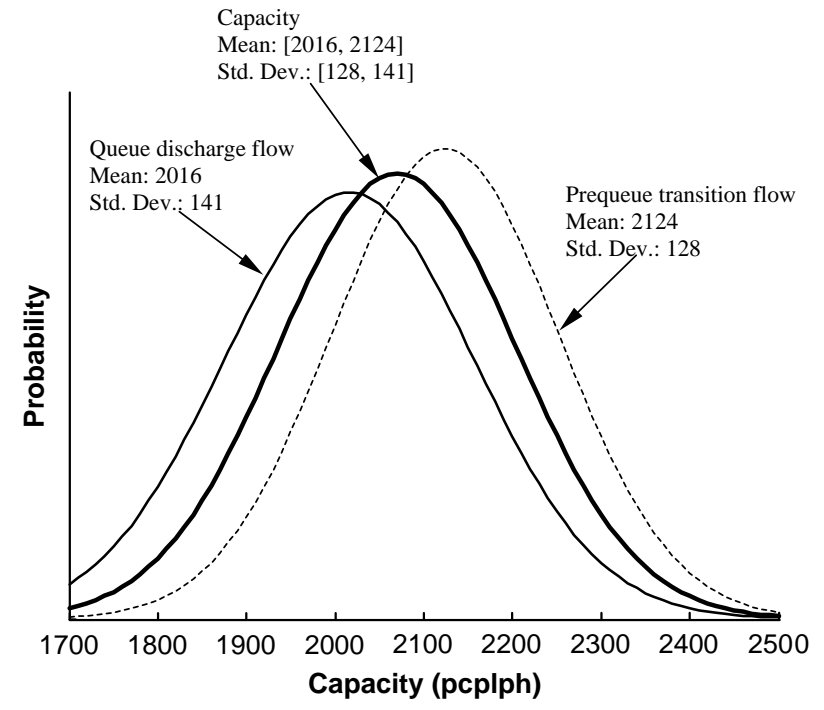

FIGURE 6 Distribution of capacity at various facilities.

Future studies should examine the factors explaining the normality and the variation of the capacity from location to location in order to improve the application of capacity analysis in freeway design, planning analysis, and even operational analysis when field measurements of the PQF and QDF are not available.

Besides the variation of capacity from location to location, the results also demonstrate that the QDFs and PQFs at the same bottleneck have high interday and intraday variation. The temporal variation of bottleneck flows influences the efficiency of real-time traffic control devices, such as ramp meters, and the accuracy of online traveler information systems. The HCM considers the temporal variation of capacity at the same bottleneck as the result of demand fluctuation, traffic composition, and driver population. Two approaches can be followed to treat the temporal capacity variation. One can study the average relationships between potential influential factors and capacity during a specific time interval (e.g., daily or 5-min capacity) so that a deterministic model adjusting capacity temporally can be applied once the quantitative features of those factors are defined and measurable. A second, probably more promising approach is stochastic modeling. The results of this study demonstrate that the daily average flows during the prequeue and the queue discharge periods can be assumed to be normal random variables. Persaud et al. $(14,15)$ found that the transition from the prequeue to the queue discharge period can also be described as a function of flows. The high variation of QDFs with the day also deserves more attention and research efforts. Future studies can explore statistical models of temporal capacity variations at freeway facilities, and such models should help engineers design efficient and reliable freeway traffic control schemes.

\section{CONCLUSIONS}

Some properties of traffic flows at active freeway bottlenecks are identified using data collected at 27 sites in the Twin Cities during a 7-week period. The diagnostic tool is an occupancy-based method with two threshold values. Any application of this method based on occupancy readings from upstream and downstream stations requires 
a careful calibration of the threshold values. Flows drop after upstream queues form at all studied bottlenecks, and the percentage flow drops at various bottlenecks range from $2 \%$ to $11 \%$, with a mean of $5.4 \%$ and a median of $4.9 \%$. Increased passage times due to acceleration noise at the bottleneck may be the reason for the flow drop $(2,3)$. The average prequeue transition period across all bottlenecks (68 $\mathrm{min} /$ peak period) was even longer than the queue discharge period (38 min/peak period) when the prequeue transition periods not followed by breakdowns were included.

The 30-s QDFs display high variation and should not be assumed to be constant. However, the daily average QDFs at each studied bottleneck seem to follow a normal distribution after two normality tests and visual inspection of the normal probability plot. Future studies could explore causes of the temporal variation of QDFs with more-sophisticated models. The variation of bottleneck flows from location to location was also examined. Though the causes are not clear, the long-run average QDFs and PQFs at studied bottlenecks both seem to be normally distributed. Therefore, capacity, defined as either the long-run PQF or the long-run QDF or a weighted sum of the two flow rates, should also follow a normal distribution. How this conclusion helps to refine the current capacity estimation procedures should be explored in future research.

If the temporal and spatial variation of capacity at various freeway bottlenecks cannot be adequately explained by measurable geometric, environmental, and human factors, a probabilistic notion of freeway capacity may be necessary in order to help engineers establish "reasonable expectation." The findings of this study suggest some directions for stochastic modeling of freeway capacity. It is also strongly recommended that traffic engineers, when performing capacity analysis on existing facilities, take full advantage of the available traffic data, such as those from loop detectors. A careful examination of the PQFs and QDFs should generate more-accurate estimates of capacity. The highest 15-min flow rate falls in the upper tail of the capacity distribution and does not represent the capacity that can be reasonably expected.

\section{REFERENCES}

1. Highway Capacity Manual. TRB, National Research Council, Washington, D.C., 2000.

2. Hall, F. L., and K. Agyemang-Duah. Freeway Capacity Drop and the Definition of Capacity. In Transportation Research Record 1320, TRB, National Research Council, Washington, D.C., 1991, pp. 91-98.

3. Banks, J. H. The Two-Capacity Phenomenon: Some Theoretical Issues. In Transportation Research Record 1320, TRB, National Research Council, Washington, D.C., 1991, pp. 234-241.

4. Banks, J. H. Two-Capacity Phenomenon at Freeway Bottlenecks: A Basis for Ramp Metering? In Transportation Research Record 1320, TRB, National Research Council, Washington, D.C., 1991, pp. 83-90.

5. Cassidy, M. J., and R. L. Bertini. Some Traffic Features at Freeway Bottlenecks. Transportation Research, Vol. 33B, 1999, pp. 25-42.

6. Zhang, L., and D. Levinson. Ramp Metering and Capacity of Active Freeway Bottlenecks. Presented at 83rd Annual Meeting of the Transportation Research Board, Washington, D.C., 2004.
7. Newman, L. Study of Traffic Capacity and Delay at the Merge of the North Sacramento and Elvas Freeways. Final Report. California Division of Highways, 1961.

8. Persaud, B. N. Study of a Freeway Bottleneck to Explore Some Unresolved Traffic Flow Issues. Ph.D. dissertation. University of Toronto, Canada, 1986.

9. Hall, F. L., and L. M. Hall. Capacity and Speed-Flow Analysis of the Queen Elizabeth Way in Ontario. In Transportation Research Record 1287, TRB, National Research Council, Washington, D.C., 1990 pp. $108-118$.

10. Persaud, B. N., and V. F. Hurdle. Freeway Capacity: Definition and Measurement Issues. Proc., International Symposium on Highway Capacity, A. A. Balkema Press, Germany, 1991, pp. 289-307.

11. Bertini, R. L., and M. J. Cassidy. Some Observed Queue Discharge Features at a Freeway Bottleneck Downstream of a Merge. Transportation Research, Vol. 36A, 2002, pp. 683-697.

12. Koshi, M., M. Kuwahara, and H. Akahane. Capacity of Sags and Tunnels on Japanese Motorways. ITE Journal, 1992, pp. 17-23.

13. Athol, P., and A. Bullen. Multiple Ramp Control for a Freeway Bottleneck. In Highway Research Record 456, HRB, National Research Council, Washington, D.C., 1973, pp. 50-54.

14. Persaud, B., S. Yagar, and R. Brownlee. Exploration of the Breakdown Phenomenon in Freeway Traffic. In Transportation Research Record 1634, TRB, National Research Council, Washington, D.C., 1998, pp. 64-69.

15. Persaud, B., S. Yagar, D. Tsui, and H. Look. Breakdown-Related Capacity for Freeway with Ramp Metering. In Transportation Research Record: Journal of the Transportation Research Board, No. 1748, TRB, National Research Council, Washington, D.C., 2001, pp. 110-115.

16. Cassidy, M. J., and J. Rudjananakanoknad. Study of Traffic at a Freeway Merge and Roles for Ramp Metering. Final Report. California Partners for Advanced Transit and Highways, University of California, Berkeley, 2002.

17. Chen, L., and A. D. May. Traffic Detector Errors and Diagnostics. In Transportation Research Record 1132, TRB, National Research Council, Washington, D.C., 1987, pp. 82-93.

18. Hall, F. L., and B. N. Persaud. Evaluation of Speed Estimates Made with Single-Detector Data from Freeway Traffic Management Systems. In Transportation Research Record 1232, TRB, National Research Council, Washington, D.C., 1989, pp. 9-16.

19. Jacobson, L. N., N. L. Nihan, and J. D. Bender. Detecting Erroneous Loop Detector Data in a Freeway Traffic Management System. In Transportation Research Record 1287, TRB, National Research Council, Washington, D.C., 1990, pp. 151-166.

20. Daganzo, C. F. Fundamentals of Transportation and Traffic Operations. Elsevier Science Inc., New York, 1997.

21. Cassidy, M. J., and J. R. Windover. Methodology for Assessing Dynamics of Freeway Traffic Flow. In Transportation Research Record 1484, TRB, National Research Council, Washington, D.C., 1995, pp. 73-79.

22. Banks, J. H. Flow Processes at a Freeway Bottleneck. In Transportation Research Record 1287, TRB, National Research Council, Washington, D.C., 1990 , pp. 20-28.

23. Das, S., and D. Levinson. A Queuing and Statistical Analysis of Freeway Bottleneck Formation. Journal of Transportation Engineering, ASCE, 2003 (in press).

24. Shapiro, S. S., and M. B. Wilk. An Analysis of Variance Test for Normality (Complete Samples). Biometrika, Vol. 52, Nos. 3 and 4, 1965 , pp. 591-611.

Publication of this paper sponsored by Highway Capacity and Quality of Service Committee. 